\title{
Autologous Hematopoietic Stem Cell Transplantation for Diffuse Large B-Cell Lymphoma
}

\author{
Eisei Kondo
}

\begin{abstract}
High-dose chemotherapy supported by autologous stem cell support/transplantation (HDT/ASCT) has been a standard of care over the last two decades in patients with relapsed or refractory(R/R) diffuse large B-cell lymphoma (DLBCL), which is sensitive to salvage chemotherapy. HDT/ASCT for high-risk DLBCL in upfront setting remains controversial, so it is not recommended for clinical practice. Various promising salvage chemotherapy regimens have been reported in phase 2 studies; however, two large randomized phase 3 studies showed similar efficacy of R-ICE vs. R-DHAP and R-GDP vs. R-DHAP. Since the registry data shows feasibility and efficacy of HDT/ASCT in elderly R/R DLBCL patients, older age ( $>65$ years) itself is not a contraindication for HDT/ASCT. Rituximab maintenance failed to demonstrate a significant benefit compared with observation only after HDT/ASCT. While sensitive R/R DLBCL might be cured by HDT/ASCT even in third-line therapy, the prognosis of insensitive R/R DLBCL is extremely poor. Further study to establish treatment strategies for high-risk patients defined by prognostic factors or biomarkers, and insensitive patients is warranted. [J Clin Exp Hematop 56(2):100-108, 2016]
\end{abstract}

Keywords: diffuse large B-cell lymphoma (DLBCL), high dose chemotharapy, autologous hematolpoietic stem cell transplantation

\section{INTRODUCTION}

Patients with relapsed or refractory $(\mathrm{R} / \mathrm{R})$ diffuse large B-cell lymphoma (DLBCL) have a poor outlook with a life expectancy of 3 to 4 months if left untreated. ${ }^{1}$ Based on the PARMA study published in 1995, high-dose chemotherapy followed by autologous stem cell transplantation/support (HDT/ASCT) has been the standard of care in R/R DLBCL patients who achieved complete remission (CR) or partial response (PR) after salvage chemotherapy (Fig. 1). ${ }^{2}$ At that time, $10 \%$ of DLBCL patients were refractory with first-line chemotherapy, and 30-40\% of DLBCL patients who responded to first-line chemotherapy relapsed in the prerituximab era, ${ }^{3}$ meaning that half of DLBCL patients proceeded to salvage chemotherapy.

The addition of rituximab $(\mathrm{R})$ to the first-line treatment (rituximab, cyclophosphamide, doxorubicin, vincristine, and prednisolone [CHOP] or CHOP-like chemotherapy) for DLBCL has resulted in a marked improvement in outcome

\section{Received: February 20, 2016}

Revised : May 4, 2016

Accepted: June 5, 2016

Department of General Medicine, Okayama University Graduate School of Medicine, Dentistry and Pharmaceutical Sciences, Okayama, Japan

Corresponding author: Eisei Kondo, M.D, Ph.D., Department of General Medicine, Okayama University Graduate School of Medicine, Dentistry and Pharmaceutical Sciences, 2-5-1, Shikata, Okayama 700-8558, Japan

E-mail: eisei-k@md.okayama-u.ac.jp for both elderly and young patients. ${ }^{1,4}$ Five-year overall survival (OS) was improved from about $50 \%$ with chemotherapy alone ${ }^{3,5}$ to $75-80 \%$ with R-containing chemotherapy. ${ }^{5,6}$ Therefore, the rate of primary refractory and relapsed patients was reduced to $4 \%$ and $10-20 \%$, respectively. ${ }^{1}$ As prognosis of DLBCL patients in whom R-CHOP failed has been reported to be worse than prognosis of those who failed to $\mathrm{CHOP}$ alone, ${ }^{5,7}$ it is difficult to improve the response rate of salvage chemotherapy in the R-era. In the randomized phase 3 trial for untreated high-risk DLBCL, the benefit of HDT/ASCT at up-front setting was shown in progression free survival, but not in overall survival. ${ }^{8}$ HDT/ASCT as consolidation therapy in first-line treatment for DLBCL is therefore not generally recommended at present. In this review, I describe recent research and advances in the treatment strategy of R/R DLBCL patients eligible for HDT/ASCT.

\section{SALVAGE CHEMOTHERAPY REGIMENS FOR R/R DLBCL}

In the PARMA study, ${ }^{2}$ the salvage chemotherapy regimen was DHAP (dexamethazone, high-dose cytarabine and cisplatin) with an overall response rate after 2 cycles of $52.7 \%$. The patients who were sensitive to the salvage chemotherapy could proceed to HDT/ASCT if they did not meet ineligible criteria. It is therefore prerequisite to increase the response rate of salvage chemotherapy to improve the prognosis of R/R DLBCL. Various chemotherapy regimens, such 

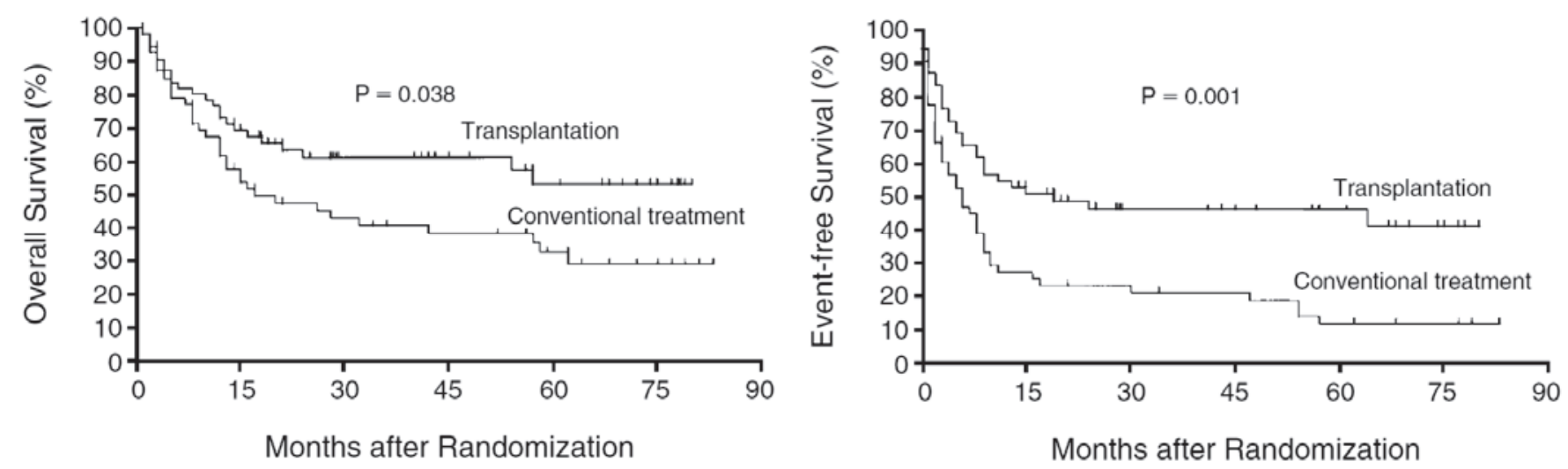

Fig. 1. Overall survival (left) and event-free survival (right) of patients in the transplantation and conventional-treatment groups in the PARMA study. (Philip T, et al., Ref. 2)

Table 1. Outcome of salvage chemotherapy regimens for relapsed or refractory aggressive lymphoma patients in phase 2 studies

\begin{tabular}{|c|c|c|c|c|c|c|c|}
\hline Regimen & $\begin{array}{c}\mathrm{n} \\
(\mathrm{DLBCL})\end{array}$ & ORR, \% & $\mathrm{CR}, \%$ & OS, $\%$ & PFS, \% & $\begin{array}{c}\text { Transplanted } \\
(\%)\end{array}$ & Reference \\
\hline DHAP & 90 & 62 & 34 & $25(2 \mathrm{y})$ & NA & NA & 9 \\
\hline ICE & $222(176)$ & 72 & 28 & $38(5 \mathrm{y})$ & $29(5 \mathrm{y})$ & $149(67 \%)$ & 24 \\
\hline R-ICE & 28 & 71 & 25 & $72(1 \mathrm{y})$ & $60(1 \mathrm{y})$ & $19(68 \%)$ & 14 \\
\hline R-ICE & $36(36)$ & 78 & 53 & $67(2 \mathrm{y})$ & NA & $25(69 \%)$ & 13 \\
\hline ESHAP & $122(46)$ & 64 & 37 & $31(3 \mathrm{y})$ & NA & NA & 25 \\
\hline \multirow[t]{2}{*}{ R-ESHAP } & $163(163)$ & Re: 67 & Re: 37 & Re: 37 (3 y) & Re: 17 (3 y) & $98(60 \%)$ & 10 \\
\hline & & Rn: 81 & Rn: 56 & Rn: 67 (3 y) & Rn: 57 (3 y) & & \\
\hline MINE & 44 & 73 & 41 & $52(2 \mathrm{y})$ & $38(2 \mathrm{y})$ & $35(77 \%)$ & 15 \\
\hline CHASER & $32(11)$ & 67 & 53 & 39.3 mon & NA & $19(59 \%)$ & 18 \\
\hline GDP & $51(40)$ & 49 & 16 & NA & NA & $22 / 35(63 \%)$ & 19 \\
\hline GEMOX-R & $32(32)$ & 43 & 34 & $41(1 \mathrm{y})$ & $29(1 \mathrm{y})$ & NA & 26 \\
\hline R-GEMOX & $46(36)$ & 83 & 50 & $66(2 \mathrm{y})$ & $43(3 \mathrm{y})$ & NA & 21 \\
\hline
\end{tabular}

Re, patients treated with rituximab in previous chemotherapy; Rn, patients not pretreated with rituximab; NA, not applicable; y, year; DLBCL, diffuse large B-cell lymphoma; ORR, overall response rate; CR, complete response; OS, overall survival; PFS, progression-free survival; DHAP, dexamethazone, high-dose cytarabine, and cisplatin; ICE, ifosfamide, calboplatin, and etoposide; R-ICE, rituximab, ifosfamide, calboplatin, and etoposide; ESHAP, etoposide, methylprednisolone, cytarabine, and cisplatin; R-ESHAP, rituximab, etoposide, methylprednisolone, cytarabine, and cisplatin; MINE, mesna, ifosfamide, mitoxantrone, and etoposide; CHASER, cyclophosphamide, high-dose cytarabine, dexamethasone, and etoposide; GDP, gemcitabine, dexamethazone, and cisplatin; GEMOX-R, rituximab, gemcitabine and oxaliplatin; R-GEMOX, rituximab, gemcitabine and oxaliplatin

as ESHAP (etoposide, methylprednisolone, cytarabine and cisplatin), ${ }^{9-11}$ ICE (ifosfamide, calboplatin and etoposide), ${ }^{12-14}$ MINE (mesna, ifosfamide, mitoxantrone and etoposide), ${ }^{15}$ IVAD (ifosfamide, etoposide, cytarabine, and dexamethasone), ${ }^{16,17}$ CHASE (cyclophosphamide, high-dose cytarabine, dexamethasone and etoposide), ${ }^{18}$ and gemcitabine containing ${ }^{19-21}$ have been explored in phase 2 studies and retrospective studies (Table 1). These regimens seemed to be promising because the overall response rate (ORR) was favorable compared with that of the DHAP regimen $(52.7 \%)$ in the
PARMA study. Another important issue in salvage chemotherapy is the efficiency of hematopoietic stem cell mobilization. Even though granulocyte-colony-stimulating factor after chemotherapy increased mobilization efficiency, mobilization failure (defined as the inability to collect two million CD $34^{+}$cells $/ \mathrm{kg}$ ) occurred in 5-15\%. ${ }^{22}$ Among these candidate regimens, the ICE regimen, with an ORR of $71-78 \%$ in the phase 2 studies, ${ }^{12-14}$ was chosen to compare with the DHAP regimen in the multicenter phase III study (CORAL study). ${ }^{23}$ 


\section{RANDOMIZED PROSPECTIVE STUDIES OF HDCT IN THE SALVAGE SETTING (1) CORAL STUDY}

The CORAL study ${ }^{23}$ was designed to evaluate the superiority of the R-ICE regimen with the R-DHAP regimen on the primary endpoint of mobilization adjusted response rate (MARR). The MARR is the composite endpoint of ORR mobilization failure, meaning the rate of patients who are able to proceed to HDT/ASCT. The MARR was estimated to be $60 \%(75 \%$ ORR - $15 \%$ mobilization failure) for R-ICE and $45 \%$ (65\% ORR - 20\% mobilization failure) for the corresponding regimen, R-DHAP. In total, $396 \mathrm{R} / \mathrm{R}$ aggressive CD20 $0^{+}$B-cell non-Hodgkin lymphoma (NHL), including DLBCL, patients were randomized to receive R-ICE or R-DHAP (Table 2). The ORR was similar for the R-ICE arm and the R-DHAP arm at $63.5 \%$ (95\% confidence interval [CI], $56.8 \%$ to $70.7 \%$ ) and $62.8 \%(95 \% \mathrm{CI}, 55.6 \%$ to $69.7 \%)$, respectively. As 20 patients $(10 \%)$ in the R-ICE arm and 15 patients $(8 \%)$ in the R-DHAP arm failed to mobilize two million $\mathrm{CD} 34^{+}$cells $/ \mathrm{kg}$, the MARR was not significantly different $(52.3 \%$ vs. $54.5 \%)$ (Table 3$)$. There was no significant difference in the 3-year event-free survival (EFS) $(26 \%$ in $\mathrm{R}$-ICE and $35 \%$ in R-DHAP; $p=0.6$ ), the 3 -year prog- ression-free survival (PFS) (31\% in R-ICE and $42 \%$ in R-DHAP; $p=0.4)$ and the 3 -year OS (47\% in R-ICE and $51 \%$ in R-DHAP; $p=0.4)$. The superiority of the R-ICE regimen was not confirmed in the phase III study.

\section{RANDOMIZED PROSPECTIVE STUDIES OF HDCT IN THE SALVAGE SETTING (2) NCIC- CTG LY.12 STUDY}

The NCIC-CTG LY.12 study ${ }^{27}$ was planned to prove the non-inferiority of the GDP (gemcitabine, dexamethasone and cisplatin) regimen compared with the DHAP regimen, which was a different approach from the CORAL study. The GDP regimen has been developed as a salvage treatment for Hodgkin lymphoma and NHL, and the phase 2 study of GDP for R/R DLBCL patients revealed a $49 \%$ ORR (16\% of CR) with low hematological toxicities. ${ }^{19}$ In addition to response rate by arm after two cycles of treatment as the primary endpoint, not only OS, EFS and toxicities, but also quality of life and economic analysis, e.g. requirement of hospitalization, were analyzed. Of 619 enrolled R/R aggressive NHL patients in the LY.12 study, 67.7\% were DLBCL (Table 2). For the intention-to-treat population, the response rate with GDP was $45.2 \%$ and with DHAP the response rate was

Table 2. Comparison of characteristics in the CORAL study and NCIC CTG LY.12 study

\begin{tabular}{|c|c|c|}
\hline & CORAL STUDY $^{23}$ & NCIC CTG LY.12 STUDY ${ }^{27}$ \\
\hline Salvage regimen & (R-)ICE vs. (R-)DHAP & (R-)GDP vs (R-)DHAP \\
\hline \multirow[t]{2}{*}{ Patients number } & \multirow[t]{2}{*}{396 (ICE 202, DHAP 194) } & 619 (GDP 319, DHAP 309) \\
\hline & & 419 (GDP 216, DHAP 203)* \\
\hline Disease & $\mathrm{R} / \mathrm{R}$ aggressive $\mathrm{CD} 20^{+} \mathrm{B}$-cell $\mathrm{NHL}$ including DLBCL & $\mathrm{R} / \mathrm{R}$ aggressive lymphoma including DLBCL \\
\hline Study design & Superiority trial & Non-inferiority trial \\
\hline First randomization & R-ICE vs R-DHAP & R-GDP vs. R-DHAP \\
\hline Second randomization & R maintenance after HDT/ASCT & R maintenance after HDT/ASCT \\
\hline HDT regimen & BEAM & Not defined (mandated by institutional policy) \\
\hline Primary endpoint & Mobilization-adjusted response rate & $\begin{array}{l}\text { Response rate by arm after two cycles of } \\
\text { treatment }\end{array}$ \\
\hline Age (y), median (range) & $55(19-65)$ & $55.5(19-74)^{*}$ \\
\hline Ann Arbor stage III-IV & 240 & $285(66 \%)^{*}$ \\
\hline Elevated LDH & 198 & $188(44 \%)^{*}$ \\
\hline Prior rituximab & 244 & $325(76 \%)^{*}$ \\
\hline \multirow[t]{2}{*}{ Relapse $<12$ mon, refractory relapse $\geq 12$ mon } & 215 & $180,133^{*}$ \\
\hline & 244 & $116^{*}$ \\
\hline
\end{tabular}

*; diffuse large B-cell lymphoma subset ${ }^{28}$

HDT, high-dose chemotherapy; LDH, lactate dehydrogenase; R-ICE, rituximab, ifosfamide, calboplatin, and etoposide; R-DHAP, rituximab, dexamethazone, high-dose cytarabine, and cisplatin; R/R, relapsed or refractory; NHL, non-Hodgkin lymphoma; DLBCL, diffuse large B-cell lymphoma; HDT/ASCT, high-dose chemotherapy supported by autologous stem cell support/transplantation; BEAM, BCNU, etoposide, cyclophosphamide, and melphalan; R-GDP, rituximab, gemcitabine, dexamethazone, and cisplatin 
Table 3. Outcome in the CORAL study ${ }^{23}$ and NCIC CTG LY.12 study ${ }^{27,28}$

\begin{tabular}{|c|c|c|c|c|c|c|c|c|c|}
\hline & & $\mathrm{N}$ & ORR (\%) & $\mathrm{CR}(+\mathrm{CRu})$ & PR (\%) & $\begin{array}{l}\text { PBSC mobilization } \\
\text { failure }(\%)\end{array}$ & $\begin{array}{l}\mathrm{HDT} / \mathrm{ASCT} \\
\text { rate }(\%)\end{array}$ & $\begin{array}{c}\text { EFS } \\
\text { (3-year) }\end{array}$ & $\begin{array}{c}\text { OS } \\
\text { (3- year) }\end{array}$ \\
\hline \multirow[t]{3}{*}{ CORAL } & R-ICE & 197 & $125(63)$ & $72(36)$ & $53(27)$ & $20(10)$ & $101(51)$ & $26 \%$ & $47 \%$ \\
\hline & R-DHAP & 191 & $120(63)$ & $75(40)$ & $45(24)$ & $15(8)$ & $105(55)$ & $35 \%$ & $51 \%$ \\
\hline & & & & & & & & $p=0.6$ & $p=0.4$ \\
\hline NCIC LY.12 & R-GDP & 220 & $103(47)$ & & & $32 / 252(13)$ & $118(54)$ & $27 \%$ (4-year) & $41 \%$ (4-year) \\
\hline (DLBCL subset) & R-DHAP & 209 & $92(44)$ & & & & $106(51)$ & & \\
\hline
\end{tabular}

ORR, overall response rate; $\mathrm{CR}$, complete response; $\mathrm{CRu}$, complete remission uncertain; $\mathrm{PR}$, partial response; PBSC, peripheral blood stem cell; ASCT, autologous stem cell support/transplantation; EFS, event-free survival; OS, overall survival; R-ICE, rituximab, ifosfamide, calboplatin, and etoposide; R-DHAP, rituximab, dexamethazone, high-dose cytarabine, and cisplatin; R-GDP, rituximab, gemcitabine, dexamethazone, and cisplatin

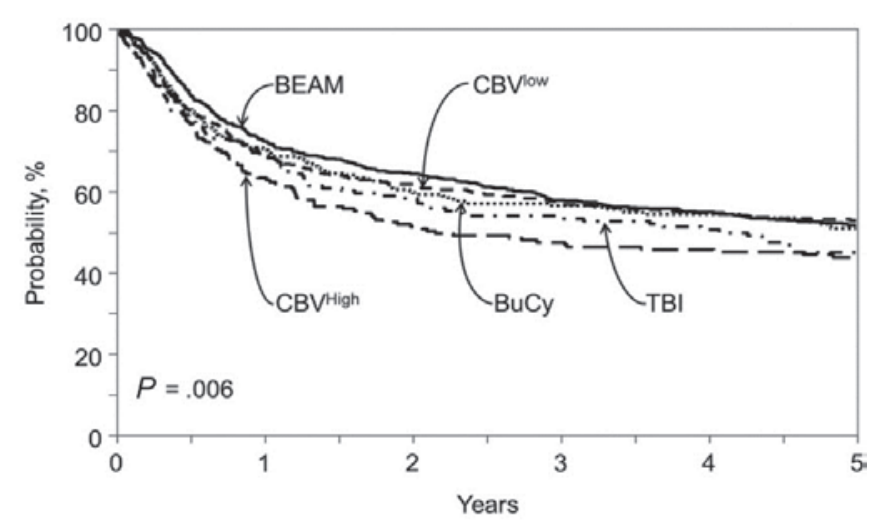

Fig. 2. Adjusted probability of overall survival after high-dose chemotherapy supported by autologous stem cell support/transplantation for diffuse large B-cell lymphoma according to conditioning regimen. (Chen YB, et al., Ref. 29)

BEAM, BCNU, etoposide, cyclophosphamide, and melphalan; CBV, cyclophosphamide, BCNU, and etoposide; BuCy, busulfan and cyclophosphamide; TBI, total body irradiation

$44.0 \%(95 \%$ CI for difference, $-9.0 \%$ to $6.7 \%)$. The onesided $95.6 \%$ upper confidence bound for each response rate was $5.7 \%$, which did not exceed the pre-specified non-inferiority margin $10 \%(p=0.005)$, meaning that the non-inferiority of GDP regimen was established. There was no significant difference in the mobilization efficiency of peripheral hematopoietic stem cells $(87.9 \%$ with GDP vs. $82.2 \%$ with DHAP, $p=0.14)$ and transplantation rate $(52.1 \%$ with GDP vs. $49.3 \%$ with DHAP, $p=0.44)$. Patients receiving GDP experienced less grade $3 / 4$ toxicity ( $47 \%$ vs. $61 \%$ ), including febrile neutropenia ( $9 \%$ vs. $23 \%$ ), platelet transfusion during the first two treatment cycles (18\% vs. 32\%) and adverse events requiring hospitalization (18\% vs. $30 \%)$.

In the DLBCL subset $(\mathrm{n}=429),{ }^{28}$ the response rate of both arms were similar (GDP 47\% (103/220 patients) vs. DHAP 44\% (92/209 patients) (Table 3). The 4-year OS and EFS in the total DLBCL group were $41 \%$ and $27 \%$, respectively. Survival was better in patients who underwent HDT/ASCT ( $n=224,4$-year OS 63\%, and EFS 46\%). The GDP regimen was non-inferior with respect to response rate to the DHAP regimen, and showed similar results for rates of transplantation and event-free survival. In addition, GDP resulted in less toxicity and was highly cost effective compared with DHAP.

\section{CONDITIONING REGIMENS OF HDT/ASCT}

The most common high-dose chemotherapy regimen used is the BEAM regimen (BCNU, etoposide, cyclophosphamide, and melphalan); however, no randomized data is available to demonstrate superiority of the BEAM regimen. The Center for International BMT Research (CIBMTR) has recently analyzed HDT regimens prior to ASCT in a total of 4917 lymphoma patients. ${ }^{29}$ In DLBCL, BEAM, CBV (cyclophosphamide, BCNU, and etoposide), BuCy (busulfan and cyclophosphamide) and total body irradiation-containing regimens were conducted in 735, 662, 279 and 161 patients, respectively. The $\mathrm{CBV}$ regimen was subdivided into $\mathrm{CBV}^{\text {low }}$ (app. $300 \mathrm{mg} / \mathrm{m}^{2}$ of BCNU; $\mathrm{n}=472$ ) and $\mathrm{CBV}^{\text {high }}$ (app. 450 $\mathrm{mg} / \mathrm{m}^{2}$ of BCNU; $\left.\mathrm{n}=190\right)$. The incidence of treatment related mortality (TRM) at 1 year was $4-8 \%$, and was not significantly different for each regimen. There was no significant difference in PFS or progression/relapse rate at 3 years among these groups. OS at 3 years was also comparable for BEAM (58\%), CBV low $(55 \%), \mathrm{BuCy}(52 \%)$ and the total body irradiation-containing regimen $(47 \%)$, but not for $\mathrm{CBV}^{\text {high }}$ (42\%) (Fig. 2). The incidence of idiopathic pneumonia syndrome, a fatal toxicity of HDT, was significantly higher in $\mathrm{CBV}^{\text {high }}$, demonstrating that $\mathrm{CBV}^{\text {high }}$ was associated with a higher mortality than BEAM (hazard ratio, 1.44, $p=$ $0.001)$ in DLBCL.

In countries and regions where BCNU is not available, various alternative HDT regimens are used. MCEC (MCNU, carboplatin, etoposide, cyclophosphamide), ${ }^{30}$ LEED (L-PAM, etoposide, cyclophosphamide, and dexa- 
methazone) $)^{31}$ and MEAM (BEAM modified regimen using MCNU) ${ }^{32}$ are common HDT regimens in Japan, and the LEAM (BEAM modified using CCNU) ${ }^{33}$ regimen was reported from India.

In an attempt to reduce the risk of common toxicity in HDT, e.g. lung injury, bendamustine instead of BCNU, with Etoposide, Cytarabine, Melphalan (BeEAM) has been developed in a phase 1-2 study. ${ }^{34}$ In forty-three patients with resistant/relapsed NHL $(\mathrm{n}=28)$ or Hodgkin lymphoma $(\mathrm{n}=$ 15) 14 patients $(33 \%)$ were in second or further CR, 22 patients $(51 \%)$ were in partial response and 7 patients $(16 \%)$ did not respond to the salvage chemotherapy. After a median follow-up of 18 months from transplantation, the outcome was good, as 35 patients $(81 \%)$ are alive and diseasefree. The TRM within 100 days was, of note, $0 \%$. The BeEAM regimen is currently being tested in a Phase 3 study with the BEAM regimen.

Addition of radioactive conjugated anti-CD20 antibody to the HDT regimen is another approach to improving the outcome in $\mathrm{R} / \mathrm{R}$ DLBCL. In a phase 3 study with either iodine-131 tositumomab plus BEAM (B-BEAM) or BEAM regimen in 224 patients with $\mathrm{R} / \mathrm{R}$ DLBCL, there was no significant difference between 2 year PFS (48.6\% vs. $47.9 \%)$ and 2 -year OS (65.6\% vs. $61 \%$ ) for BEAM and B-BEAM, respectively. The TRM within 100 days was similar in BEAM (4.1\%) and B-BEAM (4.9\%). ${ }^{35}$ In meta-analysis of ten studies with BEAM, including Yttrium-90-ibritumomab tiuxetan (Z-BEAM), the 2-year OS and PFS were $84.5 \%$ (n= $328)$ and $67.2 \%(n=285)$, respectively. Moreover, the TRM rate was $6.3 \%$ and the incidence rate of myelodysplastic syndrome was $2.5 \% .^{36}$ The meta-analysis concluded that $\mathrm{Z}$-BEAM is safe and effective as a conditioning regimen in relapsed/refractory DLBCL. ${ }^{36}$

\section{PROGNOSTIC FACTORS OF HDT/ASCT}

In the PARMA study, International Prognositc Index (IPI) at relapse $\mathrm{e}^{37}$ and early relapse (relapse within 12 months from diagnosis $)^{37}$ were identified as prognostic factors of $\mathrm{R} / \mathrm{R}$ aggressive lymphoma patients who underwent HDT/ASCT. Of note, both prognostic factors were again identified in the CORAL study. In multivariate analysis, ORR, 3-year EFS and 3 -year OS were affected by early relapse (ORR, $46 \%$ vs. $88 \%, p<0.001$; 3 -year EFS, $20 \%$ vs. $45 \%, p<0.001$; 3 -year OS, 39\% vs. $64 \%, p<0.001)$, high secondary age adjusted IPI (saaIPI) (ORR, 52\% vs. 71\%, $p<0.001 ; 3$-year EFS, $18 \%$ vs. $40 \%, p<0.001 ; 3$-year OS, $32 \%$ vs. $62 \%, p<0.001)$ and prior rituximab (ORR, 51\% vs. 83\%, $p<0.001 ; 3$-year EFS, $21 \%$ vs. $47 \%, p<0.001$; 3 -year OS, $40 \%$ vs. $66 \%, p<$ $0.01) .38$

In an attempt to understand the biologic basis for the outcomes of R/R DLBCL patients, biomarkers were analyzed as a subsequent analysis of the CORAL study. ${ }^{39}$ Using histologic material of 249 out of 396 patients in the CORAL study, the patient data were analyzed by immunochemistry for CD10, BCL6, MUM1, FOXP1, and BCL2 expression, and fluorescent in situ hybridization for BCL2, BCL6 and c-MYC breakpoints. Hans algorithm was applied to classify DLBCL into germinal center B-cell (GCB) and non-GCB subtypes. $^{40}$ By univariate analysis, MYC (8q24) gene rearrangement was the parameter to be correlated with a worse PFS $(p=0.02)$ and a worse OS $(p=0.04)$. Interestingly, patients with GCB DLBCL who were treated with R-DHAP had a better PFS than patients with non-GCB DLBCL (3-year PFS, $52 \%$ vs. $32 \%$, respectively; $p=0.01)$. In patients treated with R-ICE, there was no difference between patients with GCB and non-GCB (3-year-PFS, 31\% vs. 27\%, respectively; $p=0.8)$. Multivariate analysis has shown an independent prognostic impact of the following parameters on PFS: GCB/non-GCB Hans phenotype interaction with treatment $(p=0.04)$, prior rituximab exposure $(p=0.0052)$, saaIPI $(p=0.039)$, and FoxP1 expression $(p=0.047)$. The patients with GCB R/R DLBCL as classified by Hans algorithm seemed to benefit from the R-DHAP regimen. In interim analysis of an ongoing phase 2 study for R/R DLBCL $(\mathrm{n}=20)$, all 8 patients in early relapse (primary refractory or relapse within 1 year after initial diagnosis) did not achieve $\mathrm{CR}$ or PR, and therefore could not proceed to HDT/ASCT. ${ }^{41}$ A retrospective study has shown double-expressor lymphoma, which overexpresses MYC and BCL2 protein, and a known prognostic factor in untreated DLBCL, was also associated with poorer overall survival in R/R DLBCL. ${ }^{42}$ New treatment strategies, e.g. allogeneic HSCT, molecular targeted agent combination therapy seems to be warranted for these high-risk R/R DLBCL patients.

\section{RITUXIMAB MAINTENANCE AFTER HDT/ASCT}

In both phase III studies, CORAL and LY.12, patients who received HDT/ASCT were again randomized for either $\mathrm{R}$ maintenance or observation. In the CORAL study, 122 patients received rituximab every 2 months for one year after HDT/ASCT, and 120 patients were left for observation. ${ }^{38}$ At a median follow-up of 44 months, the 4-year EFS after HDT/ ASCT was 52\% and 53\% for the rituximab and observation groups, respectively. There was also no significant improvement of PFS (52\% vs. $53 \%)$ or OS (61\% vs. 65\%) at four years in the R maintenance group. The factors affecting EFS after HDT/ASCT were early relapse $(46 \%$ for relapsed disease within 12 months vs. $56 \%$ for relapsed disease after 12 months), high saaIPI (37\% vs. $61 \%$ ), prior treatment with rituximab (47\% vs. $59 \%)$ and male sex (46\% vs. $63 \%)$. In the multivariate analyses of PFS, saaIPI $(p<0.001)$ and male sex $(p=0.01)$ remained significant prognostic factors. Toxicity was increased by $15 \%$ and serious adverse events were reported in the rituximab arm after day 100 , the majority of 
which were infections. Interestingly, the difference in survival between female and male was observed only in the $\mathrm{R}$ maintenance arm.

The survival advantage of female sex in $\mathrm{R}$ maintenance has been repeatedly reported in the study for follicular lymphoma ${ }^{43,44}$ and DLBCL in first line, ${ }^{45,46}$ and is explainable by slower clearance of rituximab in females than in males. ${ }^{47}$

In the second randomization of the LY.12 study, the R/R CD20-positive aggressive lymphoma patients after HDT/ ASCT were assigned to rituximab $375 \mathrm{mg} / \mathrm{m}^{2}$ every 2 months for 6 doses $(n=115)$, or observation $(n=115)$. Seventeen $\%$ of the patients had transformed from previous indolent lymphoma. After a median follow-up of 63 months, 2 year EFS was $64 \%$ for the R maintenance group vs. $51 \%$ for the observation group $(p=0.11)$ and there was also no difference in 4-year OS (R 69\% vs. observation 68\%). In multivariable analysis, age $>60$ was significantly associated with EFS. Grade 3-4 neutropenia, Grade 3-4 thrombocytopenia and febrile neutropenia was observed more frequently with $\mathrm{R}$ maintenance. ${ }^{27}$

\section{INSENSITIVE R/R DLBCL PATIENTS TO SALVAGE CHEMOTHERAPY}

There is no standard treatment strategy for R/R DLBCL patients not responding to salvage chemotherapy, as the data is very limited. Van Den Neste et al. recently reported follow-up data in 145 patients who failed to proceed to HDT/ ASCT in the CORAL study. ${ }^{48}$ In the study, the third-line treatment conducted was ICE-type (19\%), DHAP-type (19\%), gemcitabine-containing (16\%), CHOP-like (8\%), dexaBEAM (8\%), and miscellaneous (31\%) regimens with or without rituximab. ORR was $43 \%$, with $29 \% \mathrm{CR} /$ complete remission uncertain $(\mathrm{CRu})$ and $14 \% \mathrm{PR} . \mathrm{CR} / \mathrm{CRu}$ and $\mathrm{PR}$ among patients treated with ICE-type, DHAP-type, gemcitabine-containing, or CHOP-like regimens were 23 and $23 \%, 35$ and $8 \%, 9$ and $4 \%, 25$ and $25 \%$, respectively. Among the 145 patients, 56 patients proceeded to HDT/ ASCT and 8 patients were treated by allogeneic hematopoietic stem cell transplantation. At median follow-up of 32.8 months, median OS, calculated from the time of second failure until death, was 5.9 months, and was not influenced by the type of the third-line regimen $(p=0.49)$. Median OS was 11.1 months in patients who were eventually underwent transplantation compared with 3.3 months in those who were not $(p<0.0001)$ (Fig. 3), corresponding to a 2-year OS of $33.9 \%$ and $9.3 \%$, respectively. HDT/ASCT is thought to have a role in in-sensitive R/R DLBCL to salvage chemotherapy, if it is sensitive to the third line treatment.

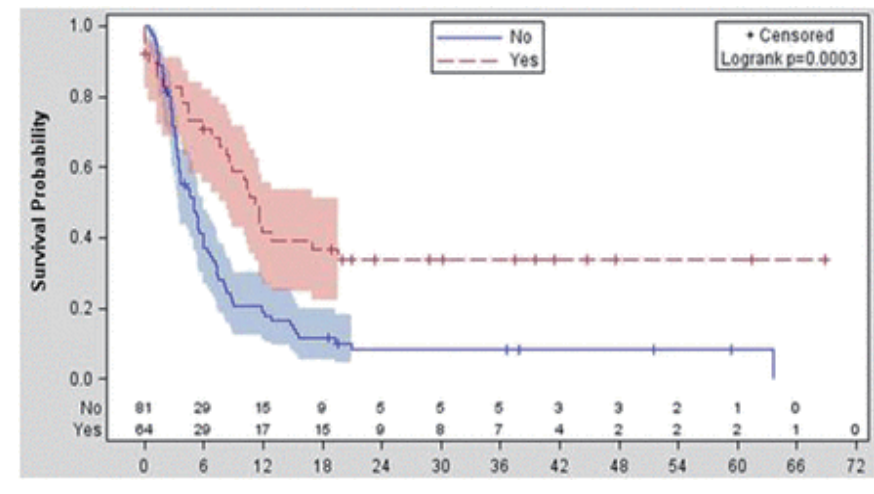

Fig. 3. Overall survival from time of treatment failure to CORAL induction according to transplantation performed (yes, $n=64$ patients; no, $\mathrm{n}=129$ patients).

(Van den Neste, et al., Ref. 48)

\section{ELIGIBILITY AGE}

Age has been thought to be a factor for eligibility of HDT/ASCT. The upper age limit for the PARMA study and the CORAL study was 60 and 65 years, respectively, and sensitive R/R DLBCL patients over 65 years will not always proceed to HDT/ASCT in practice. It was therefore unclear whether elderly patients could be treated with HDT/ASCT.

In the European Blood and Marrow Transplantation registry data, HDT/ASCT treated DLBCL patients who were $\geq$ 60 years $(n=463)$ were compared with younger patients $(<$ 60 years; $n=2,149) .{ }^{49}$ Non-relapse mortality was higher in elderly patients at 100 days $(4.4 \%$ vs. $2.8 \%, p=0.002)$, and PFS $(51 \%$ vs. $62 \%, p<0.001)$ and OS $(60 \%$ vs. $70 \%, p<$ $0.001)$ at 3 years were significantly poorer for elderly patients. Similar findings were reported from the registry of Japanese Society of Hematopoietic Cell Transplantation. ${ }^{50}$ In a total of $484 \mathrm{R} / \mathrm{R}$ DLBCL patients aged 60 years or older, who underwent HDT/ASCT, the two-year OS of patients ages 60 to 64,65 to 69 , and 70 years or older was $64.6 \%$, $50.6 \%$ and $45.7 \%$, respectively, and was significantly better in patients ages 60 to 64 years $(p=0.01)$ (Fig. 4$)$ The outcome of HDT/ASCT in elderly R/R DLBCL patients is not as good as in younger patients; however, HDT/ASCT is feasible and effective in selected elderly patients, taking the overall poorer outcome of elderly R/R DLBCL patients into account. Older age ( $>65$ years), in and of itself, is not a contraindication for autologous SCT as long as the other SCT eligibility criteria are met. ${ }^{51}$

\section{CONCLUSION}

While HDT/ASCT is still the standard of care for sensitive $\mathrm{R} / \mathrm{R} \mathrm{DLBCL}$ in the R-era, only half of $\mathrm{R} / \mathrm{R} \mathrm{DLBCL}$ patients are able to proceed to HDT/ASCT. To improve the outcome of R/R DLBCL, it is essential to identify high-risk patients by prognostic factors and biomarkers, and establish 
HDT/ASCT for DLBCL
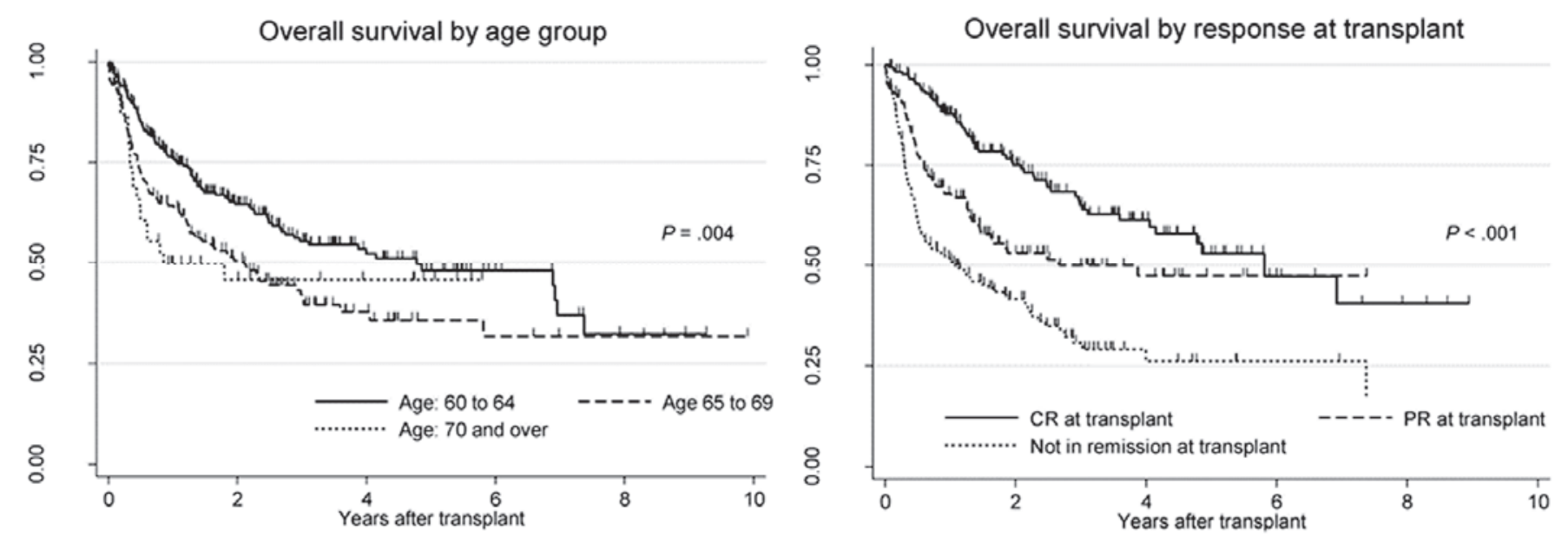

Fig. 4. Overall survival by age group (left) and overall survival by response status (right) at transplantation. (Chihara D, et al., Ref. 50) $\mathrm{CR}$, complete response; PR, partial response

treatment strategies for high-risk and insensitive patients. As the efficacy of conventional chemotherapy in high-risk patients is limited, combination with other treatment modalities, such as molecular targeted agents and allogeneic hematopoietic stem cell transplantation, may be required.

\section{DISCLOSURE STATEMENT}

The author declares that there are no conflicts of interest regarding the present paper.

\section{REFERENCES}

1 Pfreundschuh M, Trümper L, Osterborg A, Pettengell R, Trneny $\mathrm{M}$, et al.: CHOP-like chemotherapy plus rituximab versus CHOP-like chemotherapy alone in young patients with goodprognosis diffuse large-B-cell lymphoma: a randomised controlled trial by the MabThera International Trial (MInT) Group. Lancet Oncol 7:379-391, 2006

2 Philip T, Guglielmi C, Hagenbeek A, Somers R, Van der Lelie $\mathrm{H}$, et al.: Autologous bone marrow transplantation as compared with salvage chemotherapy in relapses of chemotherapy-sensitive non-Hodgkin's lymphoma. N Engl J Med 333:1540-1545, 1995

3 Fisher RI, Miller TP, O'Connor OA: Diffuse aggressive lymphoma. Hematology Am Soc Hematol Educ Program 221-236, 2004

4 Coiffier B, Lepage E, Briere J, Herbrecht R, Tilly H, et al.: CHOP chemotherapy plus rituximab compared with CHOP alone in elderly patients with diffuse large-B-cell lymphoma. $\mathrm{N}$ Engl J Med 346:235-242, 2002

5 Sehn LH, Gascoyne RD: Diffuse large B-cell lymphoma: optimizing outcome in the context of clinical and biologic heterogeneity. Blood 125:22-32, 2015
6 Zhou Z, Sehn LH, Rademaker AW, Gordon LI, Lacasce AS, et al:: An enhanced International Prognostic Index (NCCN-IPI) for patients with diffuse large B-cell lymphoma treated in the rituximab era. Blood 123:837-842, 2014

7 Chen YB, Hochberg EP, Feng Y, Neuberg D, Rawal B, et al.: Characteristics and outcomes after autologous stem cell transplant for patients with relapsed or refractory diffuse large B-cell lymphoma who failed initial rituximab, cyclophosphamide, adriamycin, vincristine, and prednisone therapy compared to patients who failed cyclophosphamide, adriamycin, vincristine, and prednisone. Leuk Lymphoma 51:789-796, 2010

8 Stiff PJ, Unger JM, Cook JR, Constine LS, Couban S, et al.: Autologous transplantation as consolidation for aggressive nonHodgkin's lymphoma. N Engl J Med 369:1681-1690, 2013

9 Velasquez WS, Cabanillas F, Salvador P, McLaughlin P, Fridrik $\mathrm{M}$, et al.: Effective salvage therapy for lymphoma with cisplatin in combination with high-dose Ara-C and dexamethasone (DHAP). Blood 71:117-122, 1988

10 Martín A, Caballero MD; Grupo Español de Linfomas/ Trasplante Autólogo de Médula Osea: R-ESHAP as salvage therapy for patients with relapsed or refractory diffuse large B-cell lymphoma: influence of prior autologous stem-cell transplantation on outcome. Haematologica 94:744, 2009

11 Soussain C, Souleau B, Gabarre J, Zouabi H, Sutton L, et al.: Intensive chemotherapy with hematopoietic cell transplantation after ESHAP therapy for relapsed or refractory non-Hodgkin's lymphoma. Results of a single-centre study of 65 patients. Leuk Lymphoma 33:543-550, 1999

12 Zelenetz AD, Hamlin P, Kewalramani T, Yahalom J, Nimer S, et al.: Ifosfamide, carboplatin, etoposide (ICE)-based second-line chemotherapy for the management of relapsed and refractory aggressive non-Hodgkin's lymphoma. Ann Oncol 14 (Suppl 1): i5-i10, 2003

13 Kewalramani T, Zelenetz AD, Nimer SD, Portlock C, Straus D, 


\section{Kondo E}

et al.: Rituximab and ICE as second-line therapy before autologous stem cell transplantation for relapsed or primary refractory diffuse large B-cell lymphoma. Blood 103:3684-3688, 2004

14 Vose J, Sneller V: Outpatient regimen rituximab plus ifosfamide, carboplatin and etoposide (R-ICE) for relapsed nonHodgkin's lymphoma. Ann Oncol 14 (Suppl 1): i17-i20, 2003

15 van Besien K, Rodriguez A, Tomany S, Younes A, Donato M, et al.: Phase II study of a high-dose ifosfamide-based chemotherapy regimen with growth factor rescue in recurrent aggressive NHL. High response rates and limited toxicity, but limited impact on long-term survival. Bone Marrow Transplant 27:397404, 2001

16 Miura K, Takei K, Kobayashi S, Kiso S, Hirabayashi Y, et al.: An effective salvage treatment using ifosfamide, etoposide, cytarabine, dexamethasone, and rituximab (R-IVAD) for patients with relapsed or refractory aggressive B-cell lymphoma. Int J Hematol 94:90-96, 2011

17 Schütt P1, Passon J, Ebeling P, Welt A, Müller S, et al.: Ifosfamide, etoposide, cytarabine, and dexamethasone as salvage treatment followed by high-dose cyclophosphamide, melphalan, and etoposide with autologous peripheral blood stem cell transplantation for relapsed or refractory lymphomas. Eur J Haematol 78:93-101, 2007

18 Oki Y, Ogura M, Kato H, Kikuchi A, Taji H, et al.: Phase II study of a salvage regimen using cyclophosphamide, high-dose cytarabine, dexamethasone, etoposide, and rituximab in patients with relapsed or refractory B-cell non-Hodgkin's lymphoma. Cancer Sci 99:179-184, 2008

19 Crump M, Baetz T, Couban S, Belch A, Marcellus D, et al.: Gemcitabine, dexamethasone, and cisplatin in patients with recurrent or refractory aggressive histology B-cell non-Hodgkin lymphoma: a Phase II study by the National Cancer Institute of Canada Clinical Trials Group (NCIC-CTG). Cancer 101:18351842,2004

20 López A, Gutiérrez A, Palacios A, Blancas I, Navarrete M, et al:: GEMOX-R regimen is a highly effective salvage regimen in patients with refractoryrelapsing diffuse large-cell lymphoma: a phase II study. Eur J Haematol 80:127-132, 2008

21 El Gnaoui T, Dupuis J, Belhadj K, Jais JP, Rahmouni A, et al.: Rituximab, gemcitabine and oxaliplatin: an effective salvage regimen for patients with relapsed or refractory B-cell lymphoma not candidates for high-dose therapy. Ann Oncol 18:1363-1368, 2007

22 Sheppard D, Bredeson C, Allan D, Tay J: Systematic review of randomized controlled trials of hematopoietic stem cell mobilization strategies for autologous transplantation for hematologic malignancies. Biol Blood Marrow Transplant 18:1191-1203, 2012

23 Gisselbrecht C, Glass B, Mounier N, Singh Gill D, Linch DC, et al.: Salvage regimens with autologous transplantation for relapsed large B-cell lymphoma in the Rituximab era. J Clin Oncol 28:4184-4190, 2010

24 Zelenetz AD, Hamlin P, Kewalramani T, Yahalom J, Nimer S, et al.: Ifosfamide, carboplatin, etoposide (ICE)-based second-line chemotherapy for the management of relapsed and refractory aggressive non-Hodgkin's lymphoma. Ann Oncol 14 (Suppl 1):i5-i10, 2003

25 Velasquez WS, McLaughlin P, Tucker S, Hagemeister FB, Swan $\mathrm{F}$, et al.: ESHAP--an effective chemotherapy regimen in refractory and relapsing lymphoma: a 4-year follow-up study. J Clin Oncol 12:1169-1176, 1994

26 López A, Gutiérrez A, Palacios A, Blancas I, Navarrete M, et al.: GEMOX-R regimen is a highly effective salvage regimen in patients with refractory/relapsing diffuse large-cell lymphoma: a phase II study. Eur J Haematol 80:127-132, 2008

27 Crump M, Kuruvilla J, Couban S, MacDonald DA, Kukreti V, et al.: Randomized comparison of gemcitabine, dexamethasone, and cisplatin versus dexamethasone, cytarabine, and cisplatin chemotherapy before autologous stem-cell transplantation for relapsed and refractory aggressive lymphomas: NCIC-CTG LY.12. J Clin Oncol 32:3490-3496, 2014

28 Kuruvilla J, MacDonald DA, Kouroukis CT, Cheung M, Olney HJ, et al:: Salvage chemotherapy and autologous stem cell transplantation for transformed indolent lymphoma: a subset analysis of NCIC CTG LY12. Blood 126:733-738, 2015

29 Chen YB, Lane AA, Logan BR, Zhu X, Akpek G, et al.: Impact of conditioning regimen on outcomes for patients with lymphoma undergoing high-dose therapy with autologous hematopoietic cell transplantation. Biol Blood Marrow Transplant 21:1046-1053, 2015

30 Kamezaki K, Kikushige Y, Numata A, Miyamoto T, Takase K, et al.: Rituximab does not compromise the mobilization and engraftment of autologous peripheral blood stem cells in diffuse-large B-cell lymphoma. Bone Marrow Transplant 39:523527,2007

31 Ogura M, Kagami Y, Taji H, Suzuki R, Miura K, et al.: Pilot phase I/II study of new salvage therapy (CHASE) for refractory or relapsed malignant lymphoma. Int J Hematol 77:503-511, 2003

32 Kawabata KC, Hagiwara S, Takenouchi A, Tanimura A, Tanuma $\mathrm{J}$, et al.: Autologous stem cell transplantation using MEAM regimen for relapsed AIDS-related lymphoma patients who received highly active anti-retroviral therapy: a report of three cases. Intern Med 48:111-114, 2009

33 Sharma A, Kayal S, Iqbal S, Malik PS, Raina V: Comparison of BEAM vs. LEAM regimen in autologous transplant for lymphoma at AIIMS. Springerplus 2:489, 2013

34 Visani G, Malerba L, Stefani PM, Capria S, Galieni P, et al.: BeEAM (bendamustine, etoposide, cytarabine, melphalan) before autologous stem cell transplantation is safe and effective for resistant/relapsed lymphoma patients. Blood 118:3419-3425, 2011

35 Vose JM, Carter S, Burns LJ, Ayala E, Press OW, et al.: Phase III randomized study of rituximab/carmustine, etoposide, cytarabine, and melphalan (BEAM) compared with iodine-131 tositumomab/BEAM with autologous hematopoietic cell 
transplantation for relapsed diffuse large B-cell lymphoma: results from the BMT CTN 0401 trial. J Clin Oncol 31:16621668,2013

36 Auger-Quittet S, Duny Y, Daures JP, Quittet P: Outcomes after (90) Yttrium-ibritumomab tiuxetan-BEAM in diffuse large B-cell lymphoma: a meta-analysis. Cancer Med 3:927-938, 2014

37 Blay J, Gomez F, Sebban C, Bachelot T, Biron P, et al:: The International Prognostic Index correlates to survival in patients with aggressive lymphoma in relapse: analysis of the PARMA trial. Parma Group. Blood 92:3562-3568, 1998

38 Gisselbrecht C, Schmitz N, Mounier N, Singh Gill D, Linch DC, et al.: Rituximab maintenance therapy after autologous stem-cell transplantation in patients with relapsed $\mathrm{CD} 20^{+}$diffuse large B-cell lymphoma: final analysis of the collaborative trial in relapsed aggressive lymphoma. J Clin Oncol 30:44624469, 2012

39 Thieblemont C, Briere J, Mounier N, Voelker HU, Cuccuini W, et al.: The germinal center/activated B-cell subclassification has a prognostic impact for response to salvage therapy in relapsed/ refractory diffuse large B-cell lymphoma: a bio-CORAL study. J Clin Oncol 29:4079-4087, 2011

40 Hans CP, Weisenburger DD, Greiner TC, Gascoyne RD, Delabie J, et al.: Confirmation of the molecular classification of diffuse large B-cell lymphoma by immunohistochemistry using a tissue microarray. Blood 103:275-282, 2004

41 Kondo E, Yamamoto K, Masunari T, Miura K, Takizawa T, et al.: Interim analysis of an ongoing phase II trial assessing safety and efficacy of R-IDEA as salvage therapy in patients with relapsed/refractory DLBCL. Ann Oncol 25 (suppl 4):iv327iv339, 2014

42 Miura K, Takahashi H, Nakagawa M, Izu A, Sugitani M, et al.: Clinical significance of co-expression of MYC and BCL2 protein in aggressive B-cell lymphomas treated with a second line immunochemotherapy. Leuk Lymphoma 57:1335-1341, 2016

43 Salles G, Seymour JF, Offner F, López-Guillermo A, Belada D, et al.: Rituximab maintenance for 2 years in patients with high tumour burden follicular lymphoma responding to rituximab plus chemotherapy (PRIMA): a phase 3, randomised controlled trial. Lancet 377:42-51, 2011
44 Jager U, Fridrik M, Zeitlinger M, Heintel D, Hopfinger G, et al.: Rituximab serum concentrations during immuno-chemotherapy of follicular lymphoma correlate with patient gender, bone marrow infiltration and clinical response. Haematologica 97:1431-1438, 2012

45 Haioun C, Mounier N, Emile JF, Ranta D, Coiffier B, et al.: Rituximab versus observation after high-dose consolidative first-line chemotherapy with autologous stem-cell transplantation in patients with poor-risk diffuse large B-cell lymphoma. Ann Oncol 20:1985-1992, 2009

46 Müller C, Murawski N, Wiesen MH, Held G, Poeschel V, et al.: The role of sex and weight on rituximab clearance and serum elimination half-life in elderly patients with DLBCL. Blood 119:3276-3284, 2012

47 Pfreundschuh M, Müller C, Zeynalova S, Kuhnt E, Wiesen MH, et al.: Suboptimal dosing of rituximab in male and female patients with DLBCL. Blood 123:640-646, 2014

48 Van Den Neste E, Schmitz N, Mounier N, Gill D, Linch D, et al.: Outcome of patients with relapsed diffuse large B-cell lymphoma who fail second-line salvage regimens in the International CORAL study. Bone Marrow Transplant 51:51-57, 2016

49 Jantunen E, Canals C, Rambaldi A, Ossenkoppele G, Allione B, et al.: Autologous stem cell transplantation in elderly patients ( $>$ or $=60$ years) with diffuse large B-cell lymphoma: an analysis based on data in the European Blood and Marrow Transplantation registry. Haematologica 93:1837-1842, 2008

50 Chihara D, Izutsu K, Kondo E, Sakai R, Mizuta S, et al.: Highdose chemotherapy with autologous stem cell transplantation for elderly patients with relapsed/refractory diffuse large B cell lymphoma: a nationwide retrospective study. Biol Blood Marrow Transplant 20:684-689, 2014

51 Oliansky DM, Czuczman M, Fisher RI, Irwin FD, Lazarus HM, et al.: The role of cytotoxic therapy with hematopoietic stem cell transplantation in the treatment of diffuse large B cell lymphoma: update of the 2001 evidence-based review. Biol Blood Marrow Transplant 17:20-47 e30, 2011 